\section{Translation of Academic Health Innovations Goes National Foundation of the Platform for Swiss Translational and Clinical Bio-Manufacturing (TCBM)}

\section{TCBM Steering Committee}

\section{Summary}

"The road to success consists in teaming up with the right partners".

Launched in September 2018, TCBM was founded by representatives of seven Swiss Universities and University Hospitals represented by five core centers in bio-manufacturing: The Basel GMP Network, the Platform for Stem Cell Research and Regenerative Medicine (SCRM) in Bern, the team in Regenerative Medicine form Fribourg, the Swiss Institute for Cell Therapies (SICT Foundation) from Geneva-Lausanne-Lugano and the Wyss Zurich. The TCBM platform supports Swiss academic biomanufacturing facilities which enable translation of promising scientific results into clinical applications.

This initiative will benefit member sites, research groups, the interaction with regulatory authorities and most importantly, the patients in terms of access to advanced and clinical grade investigational medical products. The TCBM network is further affiliated with Biotechnet Switzerland in order to promote visibility and collaboration with the private sector.

\section{Translational Research and Needs of Modern Medicine}

Academic biomedical research institutes and medical centers have a growing interest to develop their own "state-of-theart' medical products to respond to the growing need of modern medicine (ref.: SAMW publication, vol. 14, no. 1, 2019). An increasing number of therapeutic products are based on biological material (from small peptides, proteins, genes, cells to full tissues) called 'Advanced Therapeutic Medical Product' (ATMP). Similar to any medical product to be used in humans, ATMPs need to be manufactured and controlled according to quality standards (GMP, ISO, etc.) which are highly regulated by national and international laws and guidelines.

Largely handled by private industry so far, the cursor of drug manufacturing for early clinical developments has recently shifted towards academia. Indeed, on the one hand university hospitals face increasing clinical needs to provide their patients access to standard or investigative biological material (such as cells) requiring GMP capacity. On the other hand, numbers of investigators want to validate their preclinical research products at the patient's bedside (early clinical trial) as integral part of their academic study. As a result, many university research centers and hospitals have understood the overall benefit to implementing their own GMP facilities. For universities, the aspect of drug development (manufacturing and early clinical study) which was previously left to the spin-off pathway or private sector implies new choices in terms of vision, political management as well as supporting finances. Similarly, at the level of scientific and clini- cal academic investigators, taking in charge the manufacturing of their research product and performing early clinical studies also requires a commitment to dedicate part of their team and resources to 'drug development' activities (incl. new skills acquisition).

This is the field of expertise of the TCBM founding members and their platform is the first of its kind serving the academic environment.

\section{Academic Network for Bio-manufacturing}

By sharing needs, experiences and knowhow, the TCBM network aims to ease the many challenges required for GMP productions and to improve the expertise of each member to run their facilities; ultimately leading to a profit for investigators and patients alike. While many networks favor the exchange of scientific, preclinical and clinical data between researchers and clinicians, TCBM is the first Swiss nation-wide network supporting the process of bio-manufacturing and related issues such as GMP and Quality compliance.

The specific objectives are to foster the cooperation between the TCBM network partners consisting firstly in sharing expertise in operating clean rooms under good manufacturing practice and driving bio-manufacturing processes for cellular and cell-derived ATMP products for clinical studies. Secondly, TCBM member cooperation may facilitate the discussion with Swiss regulatory authorities. Finally, through networking and educational events, the platform aims to promote collaborations of research groups with the private sector potentially leading to joint applications at 'Innosuisse', the Swiss Innovation Agency, the Swiss National Science Foundation (SNSF) or even European cooperative programs.

\section{TCBM Structure and Activities}

The Steering committee (SC), composed of founding members, jointly drives the TCBM platform, currently coordinated by Prof. Eliane J. Mueller (Head of Molecular Dermatology and Stem Cell Research at University of Bern and Inselspital). An advisory board, constituted of personalities from science, industry and regulatory authorities, will be assembled ad hoc to potentiate, if necessary, the TCBM expertise.

TCBM organizes site visits, project presentations, roundtables and workshops related to GMP and other quality requirements.

\section{TCBM Illustration with Translational Projects}

Here are two examples of successful 'bench to bedside' projects from TCBM members. Beyond numerous preclinical data generated in Swiss academic laboratories, they underline the long-term interest for academic bio-manufacturing.

In Basel, the Tissue Engineering (TE) group led by Prof. Ivan Martin (Department of Biomedicine and the Department of Surgery of the University Hospital Basel) combines interdisciplinary expertise in cell biology, biomedical engineering and materials science towards the generation of human cell-based three-dimensional (3D) tissues. In a GMP setting, the TE group was able to engineer cartilage structure, derived from autologous nasal chondrocytes, for the repair of the patient articular cartilage injuries. The safety and feasibility was demonstrated in a 'firstin-man' clinical study with 18 patients. This academic phase I 


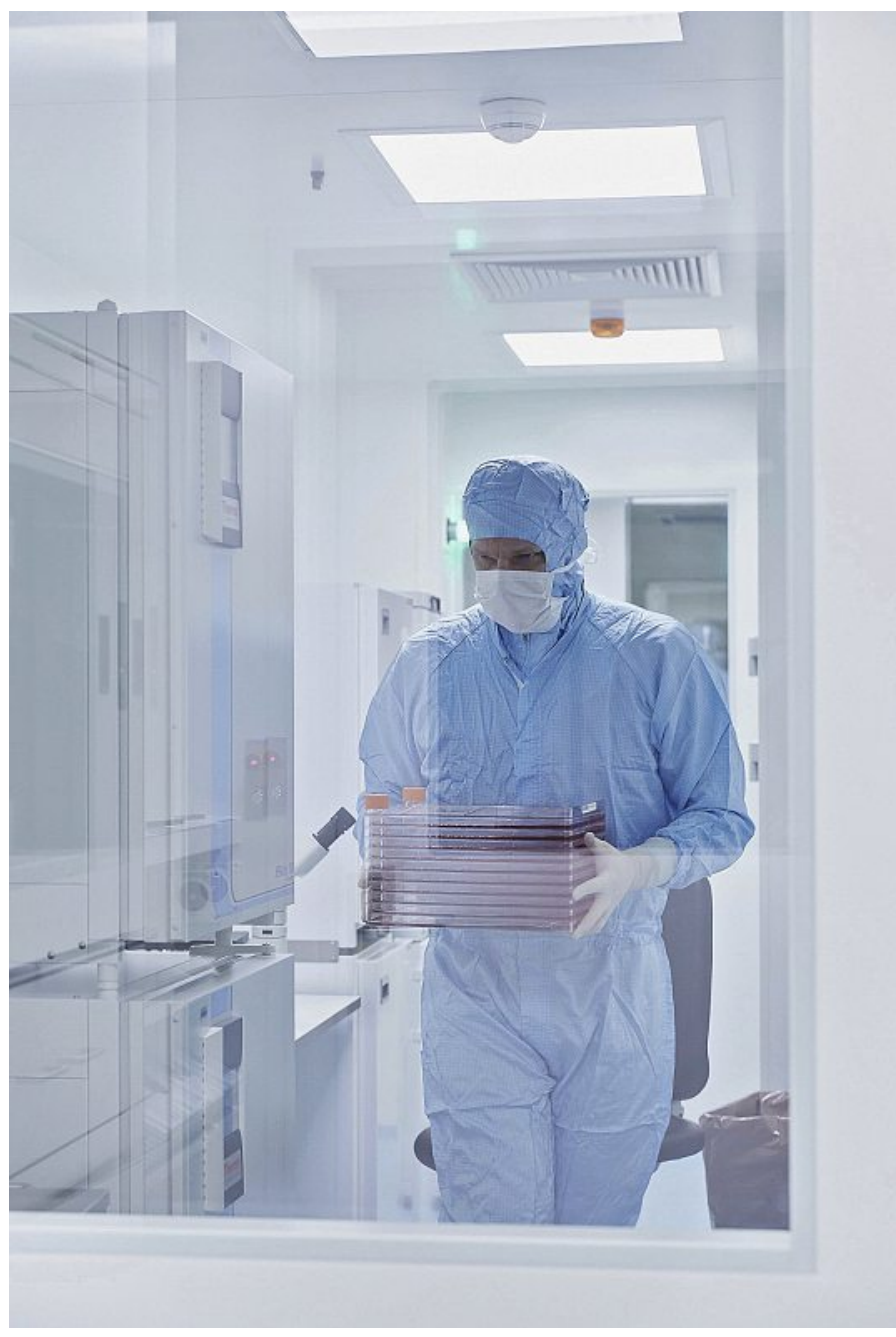

Department of Biomedicine, University of Basel, Core Facilities, Good Manufacturing Practice (GMP) Clean Room, (C) Basil Bornand

demonstration, together with funding by the Horizon 2020 program, now enabled the group to initiate an international multicenter phase II study.

In Lausanne, a cell production center (CPC) led by Dr. JeanFrançois Brunet is running at Lausanne University Hospital since 2012 and is now a functional unit of the service of pharmacy since 2018. The concept developed is the bio-confinement of the production that is done in a type of isolator that integrates incubator, microscope, working area under a supervision software to directly record all information such as physical parameters and technical data. The CPC is providing Swissmedic-approved standardized transplants to Swiss centers for burn patients as sheets of autologous keratinocytes and gives the possibility to researcher/clinician groups to develop their first phase (I/II) clinical trials in the field of cell therapy in an academic environment.

\section{TCBM Associate Members}

The TCBM platform is now opening the possibility for partners from academia, the private sector and regulatory authorities to become associate members. If you are interested in networking, workshops or further training in translational and clinical bio-manufacturing please contact the TCBM Office.

\section{The Steering Committee of the TCBM Platform:}

- Prof. Dr. PhD Eliane J. Müller; SCRM Platform, University of Bern and Inselspital and Prof. Dr. MD Gabriela Baerlocher, Department for Hematology, Inselspital, Bern.

- PD Dr. PhD Paul Zajac, Basel GMP Network, ETH-DBSSE Basel and Dr. PhD Alessia Bottos, University Hospital Basel.

- Dr. PhD Marisa Jaconi; HUG, University of Geneva, SICT Vice-Director and Dr. PhD Jean-François Brunet, Centre de production cellulaire, CHUV Lausanne, SICT vice-president.

- PD Dr. PhD Marie-Noelle Giraud, University of Fribourg and Dr. PhD Lavinia Alberi Auber, Swiss Integrative Center for Human Health, Fribourg

\section{Contact information:}

\section{TCBM Office}

René Aeberhard

University of Bern,

Department for BioMedical Research (DBMR)

Murtenstrasse 40, 3008 Bern

Telephone: +41316327912

E-mail: rene.aeberhard@dbmr.unibe.ch

TCBM Platform: https://biotechnet.ch/content/translationaland-clinical-bio-manufacturing 